\title{
Anti-attachment Function and Biomimetic Application of Slippery Zone in Nepenthes Pitcher
}

\author{
Lixin Wang' and Qiang Zhou ${ }^{2}$ \\ 1. College of Mechanical Engineering, Hebei University of Science and Technology, Shijiazhuang 050018, China \\ 2. Department of Mechatronic Engineering, China Agricultural University, Beijing 100083, China
}

\begin{abstract}
Carnivorous plants of Nepenthes species have evolved particular organs named pitchers at the tips of their conspicuous leaves, allowing slippery trapping and effective digesting prey to acquire sufficient growing nutrients. Nepenthes pitchers are generally distinguished by several morphological regions exhibiting distinct functions in prey capturing, and combined effect of the several zones results in great trapping efficiency. Depending on specific micro-structures, slippery zone performs an important role in efficiently preying arthropods, and this prey ability inspires an idea for biomimetic development of slippery trapping plate used in controlling agricultural pests. In this paper, combined with our latest results, the authors introduced the recent studies of the slippery zone, including surface structures and anti-attachment functions. They also highlighted the biomimetic application of slippery zone in developing slippery trapping plate for controlling agricultural pest.
\end{abstract}

Key words: Agricultural bionic engineering, agricultural pest, slippery trapping plate, Nepenthes pitchers, slippery surface, anti-attachment mechanism.

\section{Introduction}

Nepenthes have evolved particular organs named pitchers for effectively preying and digesting predominant arthropods, which supplies a main nitrogen and phosphorus source for surviving in infertile habitats [1, 2]. Generally, Nepenthes pitcher is recognized by several morphological regions, including a leaf-structured lid, a collar-structured peristome, a slippery zone and a digestive district [3]. Depending on specific surface architectures, the slippery zone locating between peristome and digestive region performs highly slippery function when capturing arthropods, and thereby prompts prey to be retained and slide into the beneath region $[4,5]$.

Most insects frequently result in serious damage to agricultural plants, and the generally methods for controlling agricultural pests is spraying pesticide that brings grievous environment pollution. In recent years, the mechanical approach of controlling agricultural

Corresponding author: Lixin Wang, Ph.D., research field: bionic engineering. E-mail: ck_021@tom.com. pests, which relies on insect phototaxis, light source and trapping plate, has been proposed [6-8]. This approach avoids the utilization of pesticide, thereby not only inhibiting the fearful environment pollution but also utilizing parts of the insect source as edible biological resources. Insects have evolved excellent attachment ability via natural evolution to effectively locomote and steadily stay on various substrates during taking food or escaping from being hunted. Therefore, the crucial point of mechanical controlling agricultural pests is creating efficient trapping plate, which makes the agricultural pests induced by light source to lose their normal attachment ability and slide to collection equipment. Based on this point, the slippery zone of Nepenthes pitchers presumably presents a wonderful inspiration for biomimetic development of the trapping plate.

In this present paper, combined with our latest results involving structure examinations and attachment force measurements, the authors introduced the recent research progress of the slippery zone, mainly including surface architectures and 
anti-attachment function, and the biomimetic application in developing trapping plate for controlling agricultural pest was also highlighted.

\section{Composite Structures of Slippery Zone}

Inner surface of the slippery zones in most Nepenthes pitchers appears glaucous and hydrophobic layer in macroscopic view. As being detailedly exhibited in previous literatures, this slippery surface is composed of plenty of downward-directed lunate cells and platelet-formed wax coverings forming relatively dense layer [9]. Each lunate cell orienting perpendicularly to the pitcher surface possesses an enlarged guard cell and forms a meniscoid outline with an asymmetric morphology (Fig. 1a). The wax coverings exhibiting distinct boundary consist of discernible and irregular platelet-shaped crystals, mostly overlapping each other to generate interactive architecture and arranging almost perpendicular to the subjacent tissue (Fig. 1b). Because of the existing lunate cells, the slippery surface presents obvious anisotropy to claw-structured insect attachment. These lunate cells growing in various Nepenthes species present rather different morphological feature, whereas appearance of the platelet-shaped wax crystals exhibits no noticeable difference [10].

Wax coverings of the slippery zone can be distinctly distinguished by two layers-the upper layer (Fig. 1b) and the lower layer (Fig. 1c). The upper layer consists of strikingly segregated wax platelets with the shape varying from almost ellipse to anomalistic appearance, whereas the lower layer generates an interconnected and complicated network with the platelets protruding from the beneath tissue at sharp angles and exhibits relatively much smaller dimension [11, 12]. After removing the entire wax coverings, the slippery zone exhibits a significantly smooth substrate (Fig. 1d). Wax platelets of the two layers are connected by very thin stalk-like structure [11], probably indicating that the upper wax coverings can easily detach from the adjacent layer. This structural property implies the wax crystals contribute greatly to restricting prey. In vertical direction, the platelet-shaped crystals display a sponge-like structure with fairly constant thickness of approximate $3 \mu \mathrm{m}$ [12].

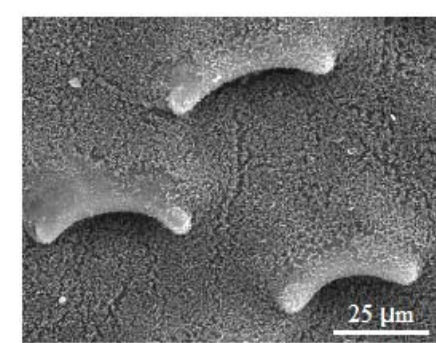

(a)

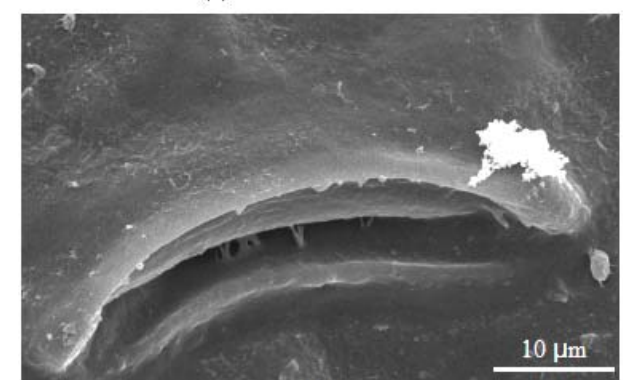

(d)

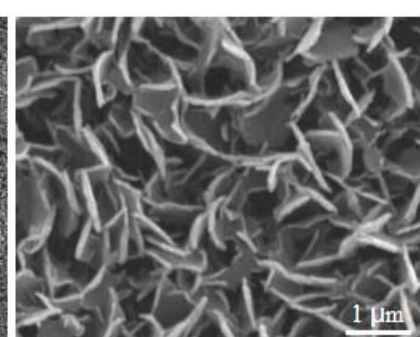

(b)

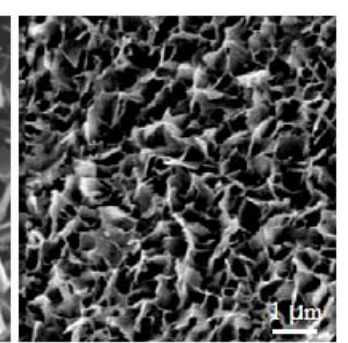

(c)

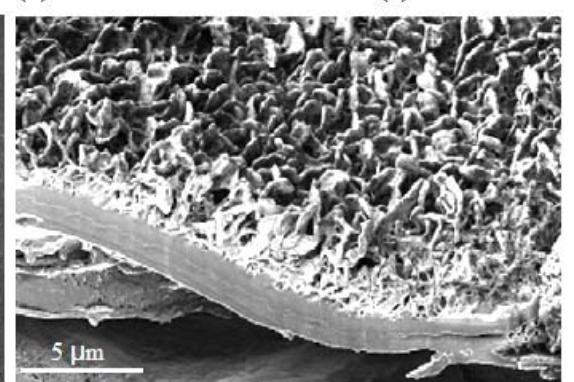

(e)

Fig. 1 Slippery region of Nepenthes pitcher: (a) Downward-directed lunate cells and irregular wax coverings; (b) platelet-shaped wax crystals from upper layer; (c) wax crystals from lower layer; (d) slippery surface when removing the wax coverings, presenting a rather smooth surface; (e) the longitudinal morphology of slippery surface, exhibiting a sponge-like structure.

c is modified after Ref. [11] and e modified after Ref. [12]. 
The sponge-like layer protrudes from a rather smooth substrate that represents the morphology of periclinal membrane (Fig. 1e).

In the slippery zones of mature Nepenthes pitchers, the largest dimension (length) of upper wax platelets is approximate $1.0 \mu \mathrm{m}$, and the lower wax crystals present slightly smaller value as $0.8 \mu \mathrm{m}$, but possess no striking different values in thickness ( $35 \mathrm{~nm}$ ) of the crystals from the two categories. However, the structural dimensions of lunate cells show great range of values that is generally greater than $10 \mu \mathrm{m}$, such as length, height and width. Without consideration of the discrimination in Nepenthes species, the distribution density of lunate cells achieves several hundred per $\mathrm{mm}^{2}$ [10, 13-15]. Our latest statistical results exhibited that the largest dimension of upper layer wax platelets in Nepenthes alata bears the values of about $1.2 \mu \mathrm{m}$, and the length and distribution density of lunate cells can reach to about $100 \mu \mathrm{m} / \mathrm{mm}^{2}$ and $266 / \mathrm{mm}^{2}$, respectively. The existing lunate cells with relatively tremendous geometric dimensions not only make the slippery surface exhibit anisotropy, but also contribute greatly to surface roughness. Observations discovered that the slippery surface is undulating and presents significant height variation (Fig. 2), leading to rather great surface roughness, the arithmetic average roughness $(R a)$ values approximately range from 1.84 $\mu \mathrm{m}$ to $3.01 \mu \mathrm{m}$ [13], which differs significantly with Scholz's result ( $R a 0.254 \mu \mathrm{m})$ [12]. This conspicuous difference probably results from the scanning area. Scholz et al. [12] examined the slippery surface within rather small area as approximate $10 \mu \mathrm{m} \times 10 \mu \mathrm{m}$, and the authors observed a relatively larger district as 160 $\mu \mathrm{m} \times 120 \mu \mathrm{m}$. Their further examination presented that the roughness value can achieve to $R a 2.653 \mu \mathrm{m}$ when including several lunate cells, but decreases significantly $(R a 0.985 \mu \mathrm{m})$ when excluding the lunate cells.

\section{Anti-attachment Mechanism of Slippery Zone}

Anti-attachment mechanism of the slippery zone serves a crucial role in biomimetic development of slippery trapping plate for controlling agricultural pests, thereby many studies have been focused on this aspect to reveal the definite mechanism. Depending on the nature evolution, insects have convergently formed two distinct attachment devices-rigid claw and adhesive pad [16]. The former can generate mechanical interlock with surface asperities, and the latter consisting of either smooth structures or hairy adhesive structures can maximize the real contact area for obtaining sufficient contact force [17, 18]. Therefore, insects mainly rely on claw and pad to generate mechanical interlock and adhesive attachment, guaranteeing effective attachment to and locomotion on various substrates. The slippery region primarily depends on its particular structures to efficiently

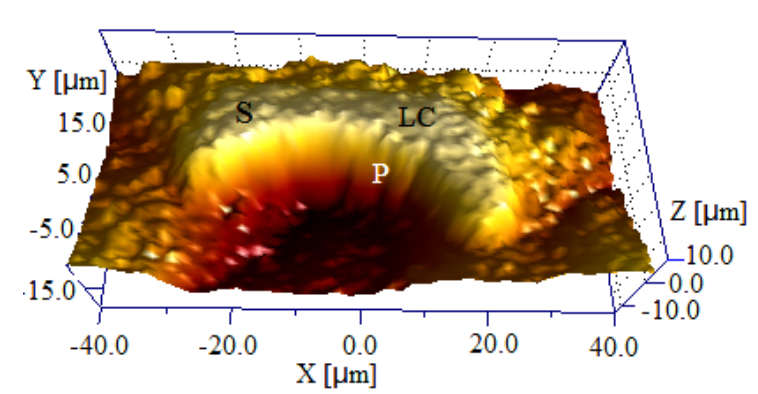

(a)

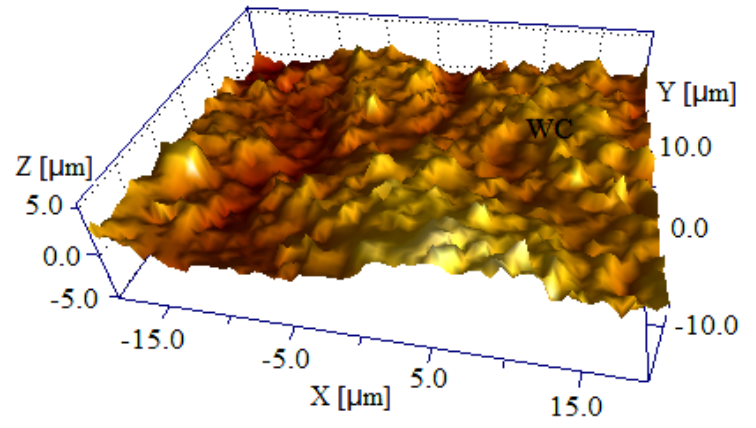

(b)

Fig. 2 3D images of slippery surface: (a) 3D view of the slippery surface surrounding the lunate cell; (b) 3D view of the wax coverings in the slippery surface (excluding the lunate cell).

LC: lunate cell; P: precipice generated by the lunate cell from upside to bottom in downward direction; S: slope generated by the lunate cell from upside to bottom in upward direction; WC: wax coverings. 
restrict insect's claws and pads to engender mechanical interlock and adhesive attachment, making the appearance of slippery behaviors.

\subsection{Effect of Wax Crystals on Insect Attachment}

Epicuticular wax crystals, which principally cover the surfaces of leaves, flowers, fruits and nonwoody stems in terrestrial plants, serve several physiological functions, including enhancing the mechanical strength, restricting uncontrollable water evaporation, decreasing contamination and pathogen attack and facilitating or prohibiting insect attachment [19]. To assess the anti-adhesive mechanism of wax crystals, numerous studies have completed composite microstructure examinations, physiochemical property analyses, behavioral observations and attachment force measurements. Based on these studies, several hypotheses have been proposed to interpret the anti-attachment properties of crystalline epicuticular waxes: (1) wax crystals are responsible for micro-roughness, which can decrease the real contact area between pad and substrate [20]; (2) wax crystals are easily detachable structures that might result in contamination to insect pad [21]; (3) the adhesive fluid secreted by insect pad, which riches in amino acids, hydrocarbons and fatty acids, interacts with wax crystals and results in slippery film [22, 23]; (4) the capillarity of the waxy structures in plant surface causes the adhesive fluid secreted by pad to be absorbed [22]. Noteworthily, all the hypotheses barely emphasized the influence of crystalline epicuticular waxes surface on insect pad, and usually ignored the influence on rigid claw.

Many laboratory experiments have presented a great accordance with the contamination hypothesis and the micro-roughness hypothesis. The climbing behaviors of flies on the slippery zone showed that crystalline wax is a key feature in the trapping system of carnivorous pitfall plants, and wax crystalloids reduced insect attachment by the contamination of insect adhesive pads [14]. Traction force testing experiments with beetles on the slippery surface of Nepenthes pitchers exhibited that the wax crystals evidently decreased the insect attachment force compared with the reference substrate, and there was no difference in the behavior and force generated by beetles on the upper or lower layer [11]. These phenomena indicated that both wax layers contributed to the trapping and retaining functions of the Nepenthes pitchers via reducing insect attachment. Presumably, the mechanical properties of wax crystals from the upper layer, such as fragile, crisp and easily breaking into tiny pieces, as well as wax crystals from the two layers, are connected by very thin stalks, providing supplementary evidence for the hypotheses of contamination.

In experiments of Scholz et al. [12], waxy fragment was not obviously detected on the pads of insect detached from mature pitcher surface. Combining with the atomic force microscopy observation on the slippery surface, they stressed that the wax crystals are mechanically stable to withstand the lateral force provided by the pads of insect, such as stick insect and ant. The microscopic roughness ( $R a 0.254 \mu \mathrm{m}$ ), root mean square roughness $(R q 0.317 \mu \mathrm{m})$ of the slippery surface was sufficient to prevent the pads not claws from attaching to the substrate. This conclusion substantiates the existence of critical surface roughness ( $R a$ 0.1-3.0 $\mu \mathrm{m}$ ), which causes conspicuous reduction of attachment ability [24, 25]; likewise it provides powerful evidence to approve the micro-roughness hypothesis and exclude the contaminations hypothesis. However, the micro-roughness hypothesis seemingly is unable to give a absolute explanation to the anti-attachment function of all Nepenthes slippery zones, because the surface roughness of slippery regions form some Nepenthes species evidently exceeds the range of critical roughness [13].

In aspect of the wax crystals' stability in slippery zones, Refs. [11, 12, 14] presented the different conclusions in some extend. Gorb' studies strongly 
confirmed the contamination hypothesis and excluded the micro-roughness hypothesis [11, 14], whereas Scholz' studies strongly supported the micro-roughness hypothesis and excluded the contamination hypothesis [12]. Further experimental studies and theoretical analysis should be conducted to confirm or exclude the existing hypotheses, or to establish an innovative hypothesis.

\subsection{Effect of Lunate Cells on Insect Attachment}

Previous studies barely focused on the wax crystals, and the lunate cell's anti-attachment function was frequently ignored. During capturing and retaining preys, the lunate cells in slippery surface may also serve the anti-attachment function. On the basis of the authors' laboratory experimental studies [9, 13], the lunate cells presumably prevent the increase of contact area mainly through the following mechanism. When locust attaches to the slippery surface, each pad of tarsus approximately occupies 173-400 lunate cells. These lunate cells with convexities and asperities presumably indicate that the slippery surface is very difficult to be duplicated, so no sufficient contact area can be acquired for locust to engender effective adhesive attachment. Besides, the wax crystals sparsely covering in the lunate cells, as well as the cavities formed by the adjacent wax platelets, probably contribute to the further reduction of the contact area. Traction force measurements confirmed that the slippery zone presented prominent anisotropy in terms of insect attachment, resulting from the particular morphology of these lunate cells. Insect claws are primarily responsible for attaching to the slippery surface. On upright direction, insects slide more effortlessly owing to the invalidation of mechanical interlock, whereas on downward direction, the insect attachment is enhanced, since the insect generate strong mechanical interlock with the inverted margins of lunate units [15].

In our current studies, results concerning the climbing behavior and friction force measurement of ants on the slippery zones, as well as micro-morphological examination and structural parameter acquisition of the slippery zones and the ant attachment organs, demonstrated that ants exhibited conspicuous differences when climbing on the upward- or downward-oriented slippery zones. Surface anisotropy of the slippery zone caused by the lunate cells is primarily responsible for these noticeable differences. A preliminary mechanical analysis confirmed that the lunate cells in natural growing direction relied on a rather small slope angle to preclude the possibility of serving as available anchorages for ant claws to generate effective attachment behaviors, thereby decreasing the attachment ability of claw-structured insect.

Although the anti-attachment mechanism of slippery zone in Nepenthes pitchers has been investigated universally, no definite hypothesis can completely reveal how the slippery zone inhibits insects' attachment ability, especially considering the dual structures of slippery zone (wax crystal, lunate cell) and attachment system (pad, claw) simultaneously. Therefore, future studies should continuously explore the anti-attachment function of slippery zone, and the expected results might present evidence to revise or exclude the former hypotheses, or establish innovative theories, as well as potentially supply theories for developing slippery trapping plates used in controlling agricultural pests.

\section{Biomimetic Applications in Developing Slippery Trapping Plate}

Nepenthes pitchers depend on their particularly evolved regions to serve the function of prey attracting, capturing and digesting for sufficient growing nutrients. In detail, mainly ascribe to special surface, it is recognized that the waxy district can effectively restrict attachment ability of insects and cause attracted preys slide to the adjacent district. This prey sliding function provides an inspiration for the biomimetic development of slippery plate, which can 
make the induced plague locusts lose their normal attachment ability, slide to particular collection equipment and consequently promote the development of mechanical controlling plague locust.

The surface morphology of many plants generates a combination of micro- and nano-hierarchical structure, representing the properties of superhydrophobic and self-cleaning [26, 27]. Their biological surface structures and specific properties can be used as models for creating biomimetic materials, and the corresponding researches have been conducted [28, 29]. Inspired by lotus leaves, various synthetic superhydrophobic self-cleaning surfaces have been prepared by creating appropriate surface morphology and roughness [30]. These research works presumably supply available methods to establish mimicking models and biomimetically create the replicas of the slippery surfaces in Nepenthes pitchers.

The fabrication of high-resolution surface replicas technique [31] probably provides the possibility to create artificial surface that mimics the topography of the slippery zones in Nepenthes pitchers. Replicas of the de-waxed slippery zone in Nepenthes pitchers have been obtained by utilizing the two-component dental wax and Spurr resin according to the two step moulding method [32]. The replicas are not seemingly suitable for acting as the slippery trapping plate used to control agricultural pests, since the dental wax or the Spurr resin is not stiff enough to withstand the penetrating of insect claw. Based on the function and structure of the slippery surface, the authors biomimetically designed and created the slippery trapping plate [33-35]. Specifically, take the scale-like expandable graphite, which possesses the relatively excellent properties of slippage and lubrication, as the substitute of the wax crystals; take the aluminum alloy plate as the substrate; and utilize the high voltage electrostatic incorporation method to create the plate. With the above substitutes and conditions, the graphite was absorbed to the substrate and tightly combined with binding medium (Fig. 3). Attachment force measurement probably demonstrated that the biomimetic plate possessed a similar anti-attachment function with the slippery zone.

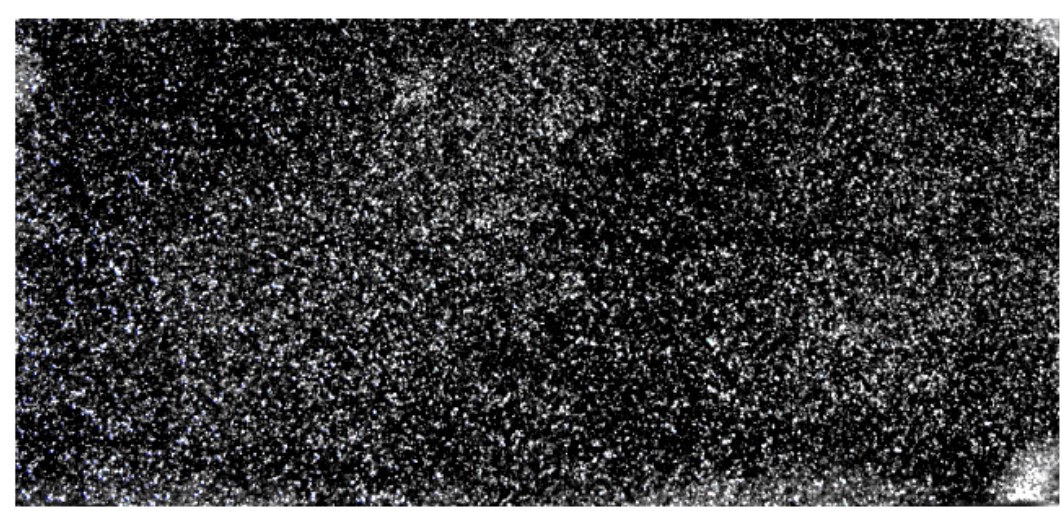

(a)

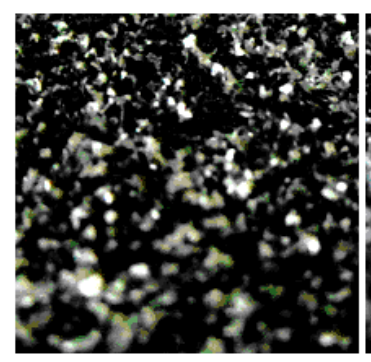

(b)

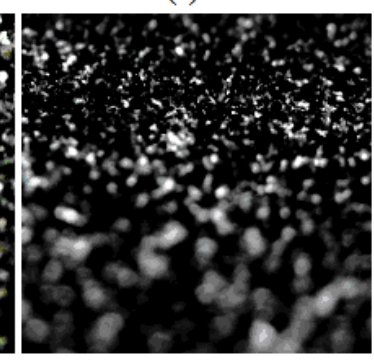

(c)

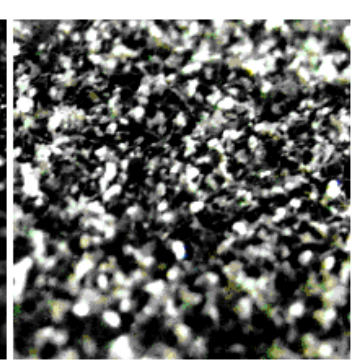

(d)

Fig. 3 Surface morphology of the biomimetic slippery trapping plate: (a) general morphology; (b-d) partial morphology. 
For biomimetically developing slippery trapping plate with excellent slippery function, the lunate cells should not be ignored, so the two step moulding method potentially provides the possibility. In future studies, large scale (length and width are about $10 \mathrm{~cm}$ ) replicas with the lunate cells created by the moulding technique may act as the substrate, and the scale-like expandable graphite can be absorbed to the substrate with the high voltage electrostatic incorporation technique. Through this processing, an innovative slippery trapping plate can be acquired and possibly present much more effective anti-attachment functions.

\section{Conclusions}

The slippery zone of Nepenthes pitchers performs a crucial role in trapping and retaining preys via effectively restricting the insect's attachment, and inspires a wonderful idea for biomimetically creating slippery trapping plate used to control agricultural pests, which can instead of the traditional method of spraying pesticides and consequently decrease the environment pollution. Based on this viewpoint, the authors introduced the morphology structures and the anti-attachment mechanism of the slippery surfaces, as well as discussed the biomimetic application of developing trapping plate. For the purpose of creating slippery trapping plate with more effective anti-attachment function, they deservedly pointed out several aspects needed to be studied in further.

\section{Acknowledgments}

The authors sincerely thank the National Natural Science Foundation of China (51205107) and the Natural Science Foundation of Hebei province (E2014208056) for their financial supports.

\section{References}

[1] Ellison, A. M., and Gotelli, N. J. 2009. "Energetics and the Evolution of Carnivorous Plants-Darwin's Most Wonderful Plants in the World.” J. Exp. Bot. 60 (1): 19-42.
[2] Thornham, D. G., Smith, J. M., Grafe, T. U., and Federle, W. 2012. "Setting the Trap: Cleaning Behavior of Camponotus schmitzi Ants Increases Long-Term Capture Efficiency of Their Pitcher Plant Host Nepenthes bicalcarata.” Func. Ecol. 26 (1): 11-9.

[3] Riedel, M., Eichner, A., and Jetter, R. 2003. “Slippery Surfaces of Carnivorous Plants: Composition of Epicuticular Wax Crystals in Nepenthes alata Blanco Pitchers.” Planta. 218 (1): 87-97.

[4] Gaume, L., Gorb, S., and Rowe, N. 2002. "Function of Epidermal Surfaces in the Trapping Efficiency of Nepenthes alata Pitchers.” New Phytol. 156 (3): 476-89.

[5] Benz, M. J., Gorb, E. V., and Gorb, S. N. 2012. "Diversity of the Slippery Zone Microstructure in Pitchers of Nine Carnivorous Nepenthes Taxa." Arthropod-Plant Interact 6: 147-58.

[6] Xu, R. Q., Zhou, Q., and Wang, S. M. 2005. "Technical Development on Mechanical Control of Locust." Transactions of the Chinese Society for Agricultural Machinery 36: 165-8.

[7] Wang, L. X., Zhou, Q., Xu, S. Y., and Niu, H. L. 2009. "Investigation of the Sliding Friction Behaviors of Locust on Slippery Plates.” Chin. Sci. Bull. 54: 4549-54.

[8] Wang, L. X., Zhou, Q., and Niu, H. L. 2010. "Influence of Photoelectric Stimulation with Different Spectrum on Sliding Friction Behaviors of Locust Oedaleus infernalis Saussure (Orthoptera: Acrididae) on Slippery Plates.” Tribol. Int. 43 (5-6): 1163-7.

[9] Wang, L. X., and Zhou, Q. 2010. "Numerical Characterization of Surface Structures of Slippery Zone in Nepenthes alata Pitchers and Its Mechanism of Reducing Locust's Attachment Force.” Adv. Nat. Sci. 3 (2): 152-60.

[10] Riedel, M., Eichner, A., Meimberg, H., and Jetter, R. 2007. "Chemical Composition of Epicuticular Wax Crystals on the Slippery Zone in Pitchers of Five Nepenthes Species and Hybrids.” Planta. 225 (6): 1517-34.

[11] Gorb, E., Hass, K., Henrich, A., Enders, S., Barbakadze, N., and Gorb, S. 2005. "Composite Structure of the Crystalline Epicuticular Wax Layer of the Slippery Zone in the Pitchers of the Carnivorous Plant Nepenthes alata and Its Effect on Insect Attachment.” J. Exp. Biol. 208: 4651-62.

[12] Scholz, I., Buckins, M., Dolge, L., Erlinghagen, T., Weth, A., Hischen, F., Mayer, J., Hoffmann, S., Riederer, M., Riedel, M., and Baumgartner, W. 2010. "Slippery Surfaces of Pitcher Plants: Nepenthes Wax Crystals Minimize Insect Attachment via Microscopic Surface Roughness.” J. Exp. Biol. 213: 1115-25.

[13] Wang, L. X., and Zhou, Q. 2011. "Friction Force of Locust Locusta migratoria manilensis (Orthoptera: 
Locustidae) on Slippery Zones Surface of Pitchers from Four Nepenthes Species.” Tribol. Lett. 44 (3): 345-53.

[14] Gaume, L., Perret, P., Gorb, E., Gorb, S., Labatd, J., and Rowea, N. 2004. "How Do Plant Waxes Cause Flies to Slide? Experimental Tests of Wax-Based Trapping Mechanisms in Three Pitfall Carnivorous Plants.” Arth. Struct. Dev. 33 (1): 103-11.

[15] Gorb, V. E., and Gorb, S. N. 2011. "The Effect of Surface Anisotropy in the Slippery Zone of Nepenthes alata Pitchers on Beetle Attachment.” Beilstein J. Nanotechnol. 2: 302-10.

[16] Federle, W., Baumgartner, W., and Holldobler, B. 2004. "Biomechanics of Ant Adhesive Pads: Frictional Forces Are Rate- and Temperature-Dependent.” J. Exp. Biol. 207: 67-74.

[17] Perez, G. P., Peressadko, A., Schwarz, H., Kastner, V., and Gorb, S. 2006. "Material Structure, Stiffness and Adhesion: Why Attachment Pads of the Grasshopper (Tettigonia viridissima) Adhere More Strongly than Those of the Locust (Locusta migratoria) (Insecta: Orthoptera).” J. Comp. Physiol. A 192 (11): 1233-43.

[18] Scholz, I., Baumgartner, W., and Federle, W. 2008. "Micromechanics of Smooth Adhesive Organs in Stick Insects: Pads Are Mechanically Anisotropic and Softer Towards the Adhesive Surface.” J. Comp. Physiol. A 194 (4): 373-84.

[19] Gorb, E. V., Voigt, D., Eigenbrode, S. D., and Gorb, S. 2008. "Attachment Force of the Beetle Cryptolaemus montrouzieri (Coccinellidae) on Leaflet Surfaces of Mutants of the Pea Pisum sativum (Fabaceae) with Regular and Reduced Wax Coverage.” Arthropod Plant Interact 2: 247-59.

[20] Gorb, E. V., and Gorb, S. N. 2008. Contact Mechanics at the Insect-Plant Interface: How Do Insects Stick and How Do Plants Prevent This? Netherlands: Springer, 243-52.

[21] Gorb, E. V., and Gorb, S. N. 2006. "Do Plant Waxes Make Insect Attachment Structures Dirty? Experimental Evidence for the Contamination Hypothesis.” In Ecology and Biomechanics: A Mechanical Approach to the Ecology of Animals and Plants, edited by Herrel, A., Speck, T., and Rowe, N. P. Boca Raton: CRC Press Inc., 147-62.

[22] Gorb, E. V., and Gorb, S. N. 2002. "Attachment Ability of the Beetle Chrysolina fastuosa on Various Plant Surfaces." Entomologia Experimentalis et Applicata 105 (1): 13-28.
[23] Votsch, W., Nicholoson, G., and Muller, R. 2002. "Chemical Composition of the Attachment Pad Secretion of Locust Locusta migratoria.” Insect Biochem. Mol. Biol. 32 (12): 1605-13.

[24] Huber, G., Gorb, S. N., Hosoda, N., Spolenakb, R., and Arzta, E. 2007. "Influence of Surface Roughness on Gecko Adhesion.” Acta Biomater. 3 (4): 607-10.

[25] Wolff, J. O., and Gorb, S. N. 2012. "Surface Roughness Effects on Attachment Ability of the Spider Philodromus dispar (Araneae: Philodromidae).” J. Exp. Biol. 215: 179-84.

[26] Priest, C., Albrecht, T. W., Sedev, R., and Ralston, J. 2009. "Asymmetric Wetting Hysteresis on Hydrophobic Microstructured Surfaces.” Langmuir 25 (10): 5655-60.

[27] Kerstin, K., Aarnoud, D., Adrian, N., Wilhelm, B., and Klaus, W. 2009. "Nanostructure of Epicuticular Plant Waxes: Self-Assembly of Wax Tubules.” Surface Science 603 (10-12): 1961-68.

[28] Nosonovsky, M., and Bhushan, B. 2007. "Biomimetic Superhydrophobic Surfaces: Multiscale Approach.” Nano Lett. 7 (9): 2633-7.

[29] Barnes, W. J. P. 2007. "Biomimetic Solutions to Sticky Problems.” Science 318: 203-4.

[30] Roach, P., Shirtcliffe, N. J., and Newton, M. I. 2008. "Progress in Superhydrophobic Surface Development." Soft Matter 4 (2): 224-40.

[31] Koch, K., Schulte, A. J., Fischer, A., Gorb, S. N., and Barthlott, W. 2008. "A Fast, Precise and Low-Cost Replication Technique for Nano- and High-Aspect-Ratio Structures of Biological and Artificial Surfaces.” Bioinspir. Biomim. 3 (4): 1943-63.

[32] Gorb. S. N. 2007. "Visualisation of Native Surfaces by Two-Step Molding.” Microscopy Today 15: 44-6.

[33] Zhou, Q., Zhou, R. W., Wang, L. X., and Niu, H. L. 2009. "Computer Simulation and Design on Bionic Slippery Microstructure Surfaces.” Transactions of the Chinese Society for Agricultural Machinery 40 (9): 201-4.

[34] Wang, L. X., Zhou, Q., and Liu, Q. H. 2011. "Dimensions of Surface Structures of Slippery Zone in Nepenthes Pitchers and Bionic Design of Locust Trapping Plate." Chinese Society for Agricultural Machinery 42 (1): 233-5.

[35] Wang, L. X., and Zhou, Q. 2011. "Function Testing of Locust Slippery Plate Manufactured Based on Waxy Zone of Nepenthes Pitchers." Chinese Society for Agricultural Machinery 42 (5): 222-5. 